\title{
The expression level of surface marker Trop2 in gastric cancer stem-like cells
}

\author{
Qi Tang ${ }^{1}$, Xingwang Kuai ${ }^{1}$,Wei Zhao ${ }^{1}$, Guipeng Ding ${ }^{1}$, Zhenning Qiu ${ }^{1}$, Zhuming Wang $^{1}$, Xiang Zou ${ }^{2 *}$ \\ ${ }^{1}$ Key Laboratory of Antibody Technique of Ministry of Health, Nanjing Medical University, Nanjing, Jiangsu 211166, China; \\ ${ }^{2}$ The Hong Kong Polytechnic University, Hong Kong, China
}

\begin{abstract}
This study aims to prepare gastric cancer stem-like cells(GCSCs) using a serum-free suspension culture, then identify it preliminarily, and observe the expression level of Trop2. Serum-free DMEM/F12 medium and lowadhesion dishes were used for suspended culture of gastric cancer (GC) cell lines MGC803. The morphological characteristics of cell spheres were observed by optical microscope; the positive rates of the CD44, CD54, EpCAM and Trop2 in MGC803 and MGC803-spheres were detected by FACS; the changes of the cell cycle in the MGC803-spheres were explored by FACS and compared with that in MGC803; the mRNA level of cancer stem cells (CSCs) regulatory genes and Trop2 in MGC803-spheres and MGC803 were detected by qRT-PCR. MGC803-spheres formed after MGC803 was cultured in serum-free medium for about 14 days; the levels of CD44, CD54, EpCAM and Trop2 in MGC803-spheres group were higher than those in MGC803 group $(P<0.05)$. The G1 phase of cell cycle in the MGC803-spheres group was obviously lower than that in MGC803 group, and the S phase of the cell cycle in the MGC803-spheres group was obviously higher than that in the MGC803 group $(P<0.05)$. The mRNA level in the CSCs regulatory genes (Oct4, Snail, Nanog) and Trop2 in MGC803-spheres group were much higher than those in MGC803 group $(P<0.05)$. MGC803-spheres could form in serum-free and suspension culture condition. The type of cells has the characteristic of CSCs, which appears in a higher proliferation state and over expression CSCs regulating genes and Trop2.
\end{abstract}

Keywords: gastric cancer, CSCs, trop2, MGC803, expression

\section{INTRODUCTION}

Gastric cancer (GC) is the fifth most common cancer and ranks as the third leading cause of cancerrelated mortality in the world ${ }^{[1,2]}$. The incidence of GC in the Asian population is the highest. Due to the insufficient understanding of the mechanism of GC and high rates of metastasis and recurrence, the prognosis of GC patients is poor, as 5-year survival rate is less than $20 \%{ }^{[3,4]}$. In recent years, some reports indicated the existence of cancer stem cells (CSCs), which pro-

*Correspondence to: Xiang Zou, The Hong Kong Polytechnic University, Hong Kong, China. E-mail: xiang.y.zou@ polyu.edu.hk. posed a new theory causing researchers to explore a new side of the cancer ${ }^{[5]}$.

CSCs are a small proportion of tumor cells, which have some features specific for stem cell, including constant self-renewal, infinite differentiation potential, high tumorigenicity and drug resistance ${ }^{[6]}$. The cell type is crucial to the occurrence and development of the tumor. Nowadays, the importance of CSCs has been realized by more and more scientists since they were first discovered in hematologic malignancy ${ }^{[7,8]}$. Some reports showed that serum-free and suspension cultured tumor cells could enrich CSCs ${ }^{[8,9]}$.

Human trophoblast cell surface glycoprotein (TACSTD2/Trop2/M1S1/GA733-1) is an oncogene, which is a $36-\mathrm{kDa}$ single-pass transmembrane pro- 
tein and was first identified in human trophoblasts. Trop2 is cleaved through TNF- $\alpha$ converting enzyme (TACE) into extracellular domain, intracellular domain, transmembrane domain and a phosphorylation of cytoplasmic tail. Intracellular domain is released from the membrane, and accumulates in the nucleus, so as to play a function. Trop2 is overexpressed in various epithelial tumors, and its expression correlates with aggressive tumor behavior ${ }^{[10-13]}$. Trop2 is also overexpressed in CSCs, and can promote cells to grow into spheres ${ }^{[9,14]}$.

Therefore, this study is intended to produce gastric cancer stem-like cells (GCSCs) by serum-free and suspension culture, preliminary identify them, and then observe the expression level and the biological characteristics of Trop2 in GCSCs.

\section{MATERIALS AND METHODS}

\section{Cell and reagents}

Human gastric cancer cell line MGC803 was purchased from KeyGEN BioTECH (Nanjing, China). DMEM/F12, DMEM, fetal bovine serum(FBS), recombinant human basic fibroblast growth factor (bFGF) and recombinant human epidermal growth factor (EGF) were purchased from Peprotech company (USA), B27 was purchased from Gibco company (USA), 6-well superlow adhesive culture dish was purchased from Corning company (USA), anti-CD44-APC, anti-CD54-PE, anti-EpCAM-FITC and isotype control were purchased from MACS company(USA). Anti-Trop2 antibody was purchased from R\&D company(USA), cell cycle analysis kit was purchased from MultiSciences(Lianke) Biotech (Hangzhou, China), TRIzol was purchased from Invitrogen company (USA), SYBRGREEN $(2 \times)$ mixture and PrimeScript ${ }^{\mathrm{TM}} \mathrm{RT}$ reagent kit were purchased from TaKaRa (Japan).

\section{Enriched GCSCs by serum-free and suspen- sion culture}

Serum-supplement medium (SSM) consisted of DMEM and $10 \%$ FBS. Serum-free medium (SFM) consisted of DMEM/F12, $20 \mathrm{ng} / \mathrm{mL}$ EGF, $10 \mathrm{ng} / \mathrm{mL}$ bFGF, and B27 $(50 \times)$. During exponential phase, GC cell line MGC803 cultured in SSM was adjusted to 5,000 cells per well and change the medium with SFM. SSM cultured MGC803 acted as the normal control group. The growth process of the MGC803 and MGC803-spheres was detected by inverted microscope.

\section{Cell cycle analysis and cell surface markers detected by fluorescence-activated cell sorter (FACS)}

The normal control MGC803 cells $\left(1 \times 10^{7} / \mathrm{mL}\right)$ were collected by trypsin digestion, while MGC803spheres were harvested and blew into single cell suspension with dropper. Part of the cells $\left(1 \times 10^{5}\right.$ cells/100 $\mu \mathrm{L}$ ) were incubated with anti-CD44-APC, anti-CD54-PE, anti-EpCAM-FITC, anti-Trop2 antibody, and isotype control, respectively (dilution $1: 100$ ), and subsequently stained using diluted FITClabeled anti-goat IgG, washed twice and re-suspended with PBS, then cell surface markers were detected by FACS Calibur Flow Cytometer (BD) Biosciences, San Jose, CA, USA). Part of the cells $\left(1 \times 10^{5}\right.$ cells $\left./ 100 \mu \mathrm{L}\right)$ were treated with $10 \mu \mathrm{L}$ PI to detect the cell cycle distribution using the FACS Calibur Flow Cytometer, according to the manufacturer's protocol.

\section{RNA extraction, reverse transcription, and quantitative PCR}

The mRNA levels of CSCs regulatory genes: Oct 4, Snail, Nanog and Trop2 were detected in the MGC803 and MGC803-spheres, respectively. RNA was extracted by TRIzol reagent and reverse transcribed into cDNA. Real-time PCR was running under the condition of $95^{\circ} \mathrm{C}$ pre-degeneration $10 \mathrm{~min}$, and with $95^{\circ} \mathrm{C}$ degeneration $15 \mathrm{~s}, 58^{\circ} \mathrm{C} 1 \mathrm{~min}, 72^{\circ} \mathrm{C}$ extending $45 \mathrm{~s}, 40$ cycles. GAP$\mathrm{DH}$ was used as internal control. The following primers were used for PCR reactions, GAPDH forward, 5'-GAAGGTGAAGGTCGGAGTC-3', reverse, 5'-GAAGATGGTGATGGGATTTC-3'; Trop2 forward, 5'-TGTCCTGATGTGATATGTCTGAG-3', and reverse, 5'-GGGTGAGAGTGGGTTGGG-3'; Oct4 forward, 5'-TTCAGCCAAACGACCATC-3', reverse, 5'-GGAAAGGGACCGAGGAGTA-3'; Snail forward, 5'-CTTCCAGCAGCCCTACGAC-3', reverse, 5'-CGGTGGGGTTGAGGATTCT-3'; Nanog forward, 5'-CGGAATTCACCATGAGTGTGGATCCAGCTTG-3', reverse 5'-ACGCGTCGACTTATCACACGTCTTCAGGTTGCATG-3’. The relative expressions of mRNA was calculated using the $\Delta \Delta \mathrm{Ct}$ method, and results were expressed as $2^{-\Delta \Delta \mathrm{Ct}}$.

\section{Statistical analysis}

The SPSS16.0 statistical software package (SPSS Inc., Chicago, IL) was used for general statistical analysis. The differences between groups were estimated by ANOVA. Values of $P$ less than 0.05 were considered statistically significant.

\section{RESULTS}

\section{Cultivates and collects GCSCs}

In this study, we selected gastric cancer cell lines MGC803 as our experimental cell lines. MGC803 cells $(5,000$ per well) were cultured in the 6 -well 
super-low adhesive culture plate, with SFM and $20 \mathrm{ng} / \mathrm{mL}$ EGF, $10 \mathrm{ng} / \mathrm{mL}$ bFGF and B27 $(50 \times)$ at $37{ }^{\circ} \mathrm{C}$ in a $5 \% \mathrm{CO}_{2}$ humidified atmosphere. Cell spheres were found after 7 days, and $14 \mathrm{~d}$ later, there were more cell spheres formed and the cell spheres grown bigger. The cell spheres formation and growing were shown in Fig.1.

\section{The expression of GCSCs surface markers detected by FACS}

The expression of CD44, CD54, EpCAM, and Trop2 in cell surface was detected by FACS.

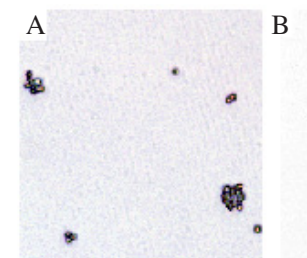

B

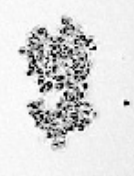

C

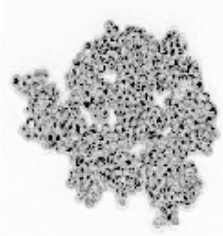

Fig. 1. GCSCs spheres. A: MGC803 cultured in SFM for 3 days, a small amount of cells aggregation and suspension was observed by inverted microscope $(\times 400)$. B: MGC803 cultured in SFM for 7 days, spheres formed $(\times 400)$. C: MGC803 cultured in SFM for 14 days, cell spheres growths like grapes $(\times 400)$.
The results indicated that the positive rates of CD44, CD54, EpCAM and Trop2 in MGC803spheres were $48.30 \% \pm 2.94 \%, 71.20 \% \pm 3.12 \%$, $7.35 \% \pm 2.39 \%$ and $44.00 \% \pm 3.18 \%$, respectively, which were higher than those in MGC803 group $(9.60 \% \pm 1.07 \%, 11.50 \% \pm 1.55 \%, 3.61 \% \pm 0.09 \%$ and $11.90 \% \pm 1.05 \%$ ), the differences are statistically significant (Fig. 2). These results indicated the GCSCs surface marker expressed in MGC803spheres were higher than those in normal MGC803.

\section{Cell cycle distribution detects by FACS}

Next, we examined the cell cycle distribution in MGC803 and MGC803-spheres by FACS. Compared with SSM cultured MGC803 group, SFM cultured MGC803-spheres group showed cell cycle arrest in $\mathrm{S}$ phase, the percentages of the G1, G2 and S phase were $45.58 \%, 7.73 \%, 46.69 \%$ in MGC803-spheres, and $53.18 \%, 7.25 \%, 39.57 \%$ in MGC803 (Fig. 3). We found that the percentage of Gl phase cell from the MGC803-spheres group was obviously lower than that from the MGC803 group, and the percentage of S phase cell from MGC803-spheres group was obvi-
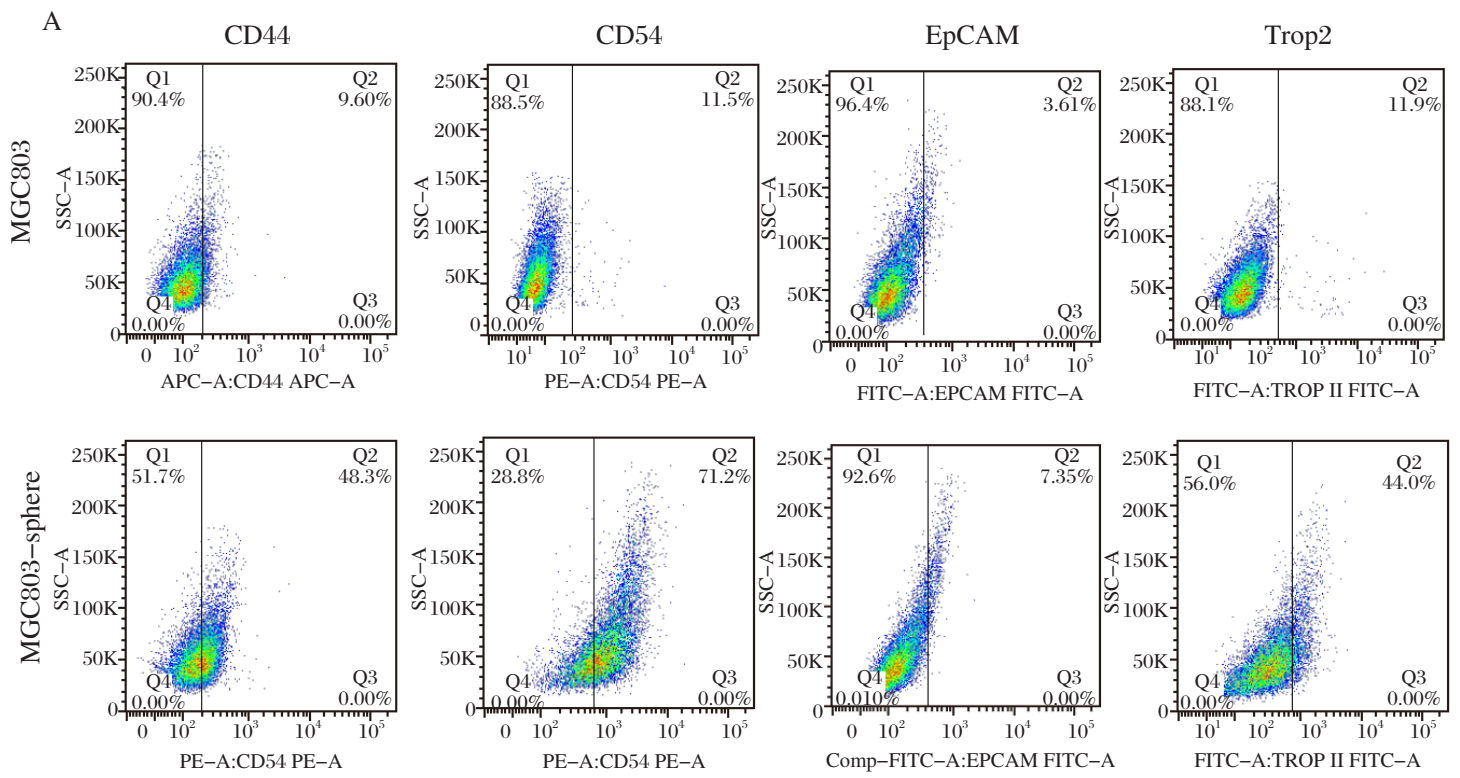

B

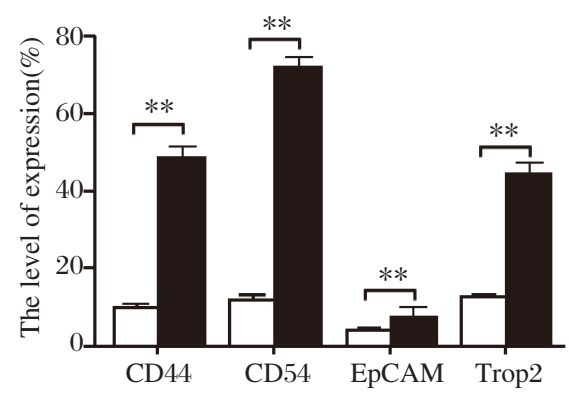

Fig. 2. The expression level of surface markers and Trop2 in MGC803 and MGC803-spheres detects by FACS. A: the expression level of CD44, CD54, EpCAM and Trop2 in MGC803 and MGC803-spheres detects by FACS. B: statistical diagram of the above results, ${ }^{* *} P<0.001$. 
ously higher than that from MGC803 group $(P<0.05)$. These results indicated that MGC803-spheres were in a high proliferation state.

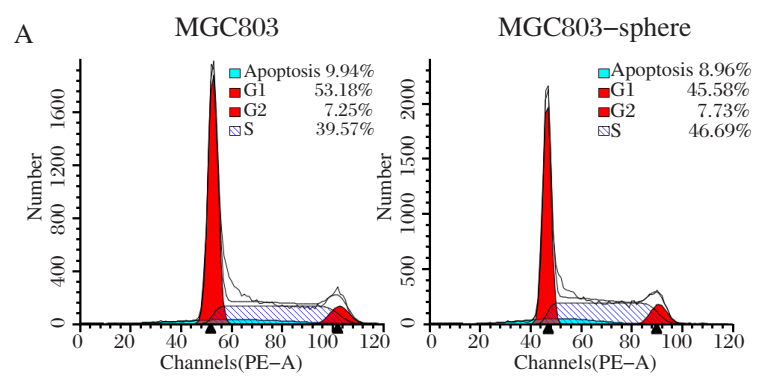

B

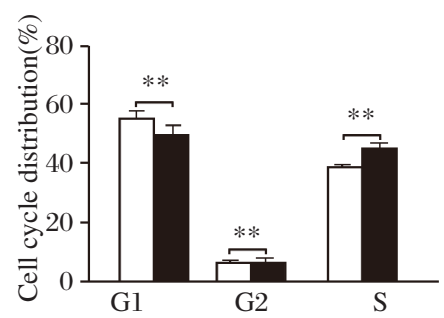

Fig. 3. Cell cycle distributions in MGC803 and MGC803spheres detects by FACS. A: Cell cycle distributions in MGC803 and MGC803-spheres detects by FACS. B: statistical diagram of the above results, ${ }^{* *} P<0.001$.

\section{The expression level of CSCs regulatory genes and Trop2 detects by qRT-PCR}

Last, we compared the mRNA levels of CSCs regulatory genes (Oct4, Snail, Nanog) and Trop2 between MGC803 and MGC803-spheres group by qRT-PCR. We found that the expression levels of Oct4, Snail, Nanog and Trop2 in MGC803-spheres were $2.30 \pm 0.13,1.90 \pm 0.17,2.50 \pm 0.11,2.90 \pm 0.15$ times of those in the MGC803 group, the differences were statistically significant (Fig.4). These results indicated CSCs regulatory genes and Trop2 were higher expressed in the MGC803-sphere.

\section{DISCUSSION}

The incidence of GC is the highest in China, which

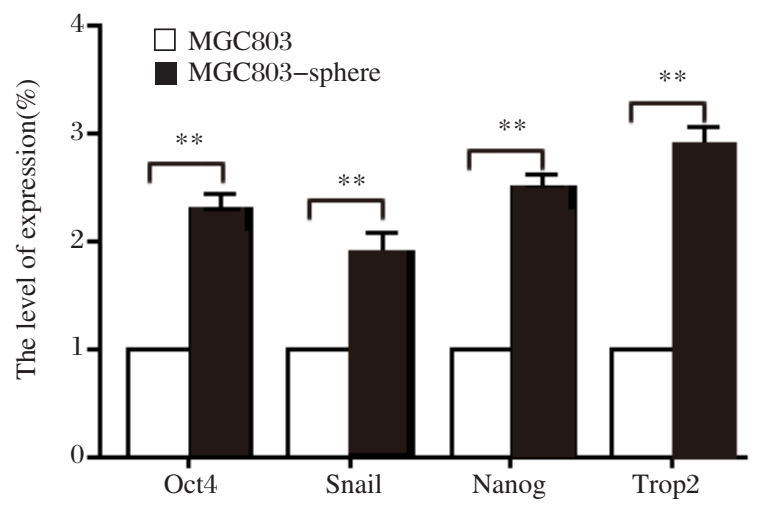

Fig. 4. The expression level of GCSCs regulatory genes and Trop2 in MGC803 and MGC803-spheres detects by qRT-PCR. ${ }^{* *} P<0.001$. ranks as the second cancer in men after lung cancer, and ranks as the fourth in women. With improved health conditions and dietary habits, the incidence of GC has declined in recent years. However, high rates of metastasis and recurrence make the prognosis of GC patients poor: 5-year survival rates are less than $20 \%{ }^{[15]}$. Therefore, it is still necessary to explore the mechanism of the invasion and metastasis of GC, and further to improve the prognosis and survival rate of GC patients. Some reports indicated that many genes have regulatory effects towards the invasion and metastasis of GC, and targeted therapy towards these genes had already made some progress ${ }^{[2,16,17]}$. Because of the complexity of the tumor formation, interaction between multiple genes, as well as the limitations of targeted drug carrier and so on, it is still urgent to find new therapeutic targets of $\mathrm{GC}$ and provide a reliable theoretical basis for medical treatment ${ }^{[18,19]}$.

Tritium-labeling studies were performed on many types of blood cancers in the 1960s, and showed the existence of a subset of primitive-appearing cells with cycling properties different from the majority of tumor cells ${ }^{[20]}$. These results coupled with earlier genetic studies indicated that many leukemias contained an immature cell population that could generate postmitotic progeny and predicted the existence of leukemic stem cell ${ }^{[16]}$. These cells, which were successfully isolated from acute myeloid leukemia and carried on a surface marker $\mathrm{CD} 34^{+} / \mathrm{CD}^{-} 8^{-}$, were injected into immunodeficient mice, and the same disease was replicated ${ }^{[21]}$. Since then, CSCs have been found in breast cancer $^{[22]}$, brain cancer ${ }^{[23]}$, prostate cancer ${ }^{[24]}$, lung cancer $^{[25]}$, colon cancer ${ }^{[26]}$, liver cancer ${ }^{[27]}$, pancreatic cancer $^{[28]}$, head and neck cancer ${ }^{[29]}$, and other solid cancers. The study of GCSCs started later, Takassi $S^{[30]}$. reported that GC cells, which were cultured in a serum-free medium with EGF and FGF, selectively expressed CD44 on the cell surface. A few weeks later, these GC cells formed spheres. After a small number of cell-spheres were injected into MOD/SCID mouse, tumor formed. Therefore, the selection of the specific cells with surface marker is very important for the identification and analysis of the GCSCs.

The main methods for separating and culturing of GCSCs are as follows, (1) surface marker selecting methods, including CD $44^{+}, \mathrm{CD}^{+} 0^{+[31]}, \mathrm{EpCAM}^{+[32]}$, $\mathrm{CD} 44^{+} \mathrm{CD} 24^{+[33]}, \mathrm{CD} 44^{+} \mathrm{CD} 54^{+[34]}$, and so on; (2) SP cell selecting method, for the cancer cells having the ability of the remove fluorescent dyes from the outside ${ }^{[35]}$; (3) SFM culture cell-spheres ${ }^{[30]}$; (4) chemotherapy drugs enriched cells ${ }^{[36]}$.

Some reports suggested that the cytoplasmic tail of Trop2 could be phosphated by protein kinase C (PKC), 
and may have different functions depending on different partners, including Claudin 1, Claudin 7, Cyclin D1, PKC, PIP2, and so on ${ }^{[9,37]}$. It has been reported that Trop2 was highly expressed in prostate cancer, and closely related to the surface marker CD133 in $\mathrm{CSCs}^{[38]}$. Our lab has been studying Trop2 since 2009, and early experimental results indicated a high expression of Trop2 in many solid tumors ${ }^{[13,39,40]}$. Subsequently we prepared several anti-Trop2 antibodies, including human anti-Trop2 antibody $\operatorname{IgG}$, fragment Fab and so on. These antibodies have been found to have the ability of binding to specific antigen-Trop2, and inhibit the proliferation, migration and invasion of the tumor cells in vivo and in vitro ${ }^{[41]}$.

CSCs have an important role in development, metastasis and invasion of cancers. In this study, we used the spheres cultured method to develop GCSCs, and selected CD $44^{[33]}, \mathrm{CD} 54^{[30]}, \mathrm{EpCAM}^{[34]}$ as the GCSCs surface markers. To observe the expression of these cell surface markers, the distribution of cell cycle and the expression levels of the CSCs regulatory genes, including Oct4, Snail, Nanog and Trop2, we selected DMEM/F12 medium with EGF and bFGF, and a super-low adhesive culture dish for the serum-free suspension culture of GC cell line MGC803 spheres. After 14 days, the cell-spheres of MGC803 were detected by inverted microscope, and the result coincide with the research of Takaishi S. on GCSCs ${ }^{[30]}$. Through FACS, we found the expression level of CD44, CD54, EpCAM in MGC803-spheres were higher than those in MGC803, and the differences were statistically significant. The results confirmed that GCSCs could be induced by serum-free and suspension method. We also found that the level of Trop2 expressed in MGC803-spheres was increased, and this indicated that Trop2 may be highly expressed in GCSCs. The examination of cell cycle distribution in MGC803-spheres showed that the S phase of MGC803-spheres was significantly higher than that in normal MGC803. This suggests that MGC803spheres have been in a high proliferation state, which is consistent with the characteristics of CSCs including self-renewal, infinite differentiation potential, etc. Some regulatory genes, such as Oct4, Sox2, Nanog, Snail, etc., are important in maintaining the pluripotent and self-renewing properties of CSCs ${ }^{[20]}$. In this study, qRT-PCR results showed that the expression levels of the CSCs regulatory genes and Trop2 in MGC803-spheres were higher than those in normal MGC803. Trop2, as an important molecule, is involved in promoting tumor development. Its roles and the mechanism in CSCs need to be studied further.

\section{Acknowledgments and funding}

This work was supported by grants from the $\mathrm{Na}-$ tional Natural Science Foundation of China (No. 81601618) and is the Innovation of the Graduate Student Training Project of Jiangsu Province (No. KYLX15_0940).

\section{References}

[1] Van Cutsem E, Sagaert X, Topal B, et al. Gastric cancer. Lancet, 2016;388:2654-64.

[2] Sue S, Shibata W, Maeda S. Helicobacter pyloriInduced signaling pathways contribute to intestinal metaplasia and gastric carcinogenesis. Biomed Res Int, 2015;2015:737621.

[3] Liu L, Chen Q, Lai R, et al. Elevated expression of mature miR-21 and miR-155 in cancerous gastric tissues from Chinese patients with gastric cancer. J Biomed Res, 2010;24:187-97.

[4] Sun R, Wang X, Zhu H, et al. Prognostic value of LAMP3 and TP53 overexpression in benign and malignant gastrointestinal tissues. Oncotarget, 2014;5:12398409.

[5] Mashima T. Cancer stem cells (CSCs) as a rational therapeutic cancer target, and screening for CSC-targeting drugs. Yakugaku Zasshi(in Japanese), 2017;137:129-32.

[6] Choi HJ, Park JH, Park M, et al. UTX inhibits EMTinduced breast CSC properties by epigenetic repression of EMT genes in cooperation with LSD1 and HDAC1. Embo Rep, 2015;16:1288-98.

[7] Rinkenbaugh AL, Baldwin AS. The NF-kappaB pathway and cancer stem cells. Cells, 2016;5(2):16

[8] Singh SR. Gastric cancer stem cells: A novel therapeutic target. Cancer Lett, 2013;338:110-9.

[9] Shvartsur A, Bonavida B. Trop2 and its overexpression in cancers: Regulation and clinical/therapeutic implications. Genes Cancer, 2015;6:84-105.

[10] Cubas R, Li M, Chen C, et al. Trop2: A possible therapeutic target for late stage epithelial carcinomas. Biochim Biophys Acta, 2009;1796:309-14.

[11] Lin JC, Wu YY, Wu JY, et al. TROP2 is epigenetically inactivated and modulates IGF-1R signalling in lung adenocarcinoma. Embo Mol Med, 2012;4:472-85.

[12] Wanger TM, Dewitt S, Collins A, et al. Differential regulation of TROP2 release by $\mathrm{PKC}$ isoforms through vesicles and ADAM17. Cell Signal, 2015;27:1325-35.

[13] Zhao W, Zhu H, Zhang S, et al. Trop2 is overexpressed in gastric cancer and predicts poor prognosis. Oncotarget, 2016;7:6136-45.

[14] Vidmar T, Pavsic M, Lenarcic B. Biochemical and preliminary X-ray characterization of the tumor-associated calcium signal transducer 2 (Trop2) ectodomain. Protein Expr Purif, 2013;91:69-76.

[15] Yoon H, Kim N. Diagnosis and management of high risk group for gastric cancer. Gut Liver, 2015;9:5-17.

[16] Makino S. The role of tumor stem-cells in regrowth of the tumor following drastic applications. Acta Unio Int 
Contra Cancrum, 1959;15(Suppl 1):196-8.

[17] Wei S, Wang L, Zhang L, et al. ZNF143 enhances metastasis of gastric cancer by promoting the process of EMT through PI3K/AKT signaling pathway. Tumour Biol, 2016;37:12813-21.

[18] Matsueda K, Toyokawa T, Ueda Y, et al. Two cases of unresectable advanced HER2-positive gastric cancer perforation during chemotherapy with trastuzumab. Nihon Shokakibyo Gakkai Zasshi(in Japanese), 2017;114:59-68.

[19] Wang Y. Transcriptional regulatory network analysis for gastric cancer based on mRNA microarray. Pathol Oncol Res, 2017; doi:10.1007/s 12253-016-0159-1.

[20] Clarke MF, Dick JE, Dirks PB, et al. Cancer stem cells-perspectives on current status and future directions: AACR workshop on cancer stem cells. Cancer Res, 2006;66:9339-44.

[21] Kassem NM. Cancer stem cells: From identification to eradication. J Egypt Natl Canc Inst, 2008;20:209-15.

[22] Thomas D, Thiagarajan PS, Rai V, et al. Increased cancer stem cell invasion is mediated by myosin IIB and nuclear translocation. Oncotarget, 2016;7:47586-92.

[23] Safa AR, Saadatzadeh MR, Cohen-Gadol AA, et al. Glioblastoma stem cells (GSCs) epigenetic plasticity and interconversion between differentiated non-GSCs and GSCs. Genes Dis, 2015;2:152-63.

[24] Jin M, Zhang T, Liu C, et al. MiRNA-128 suppresses prostate cancer by inhibiting BMI-1 to inhibit tumorinitiating cells. Cancer Res, 2014;74:4183-95.

[25] Park E, Park SY, Sun PL, et al. Prognostic significance of stem cell-related marker expression and its correlation with histologic subtypes in lung adenocarcinoma. Oncotarget, 2016;7:42502-12.

[26] Kemper K, Versloot M, Cameron K, et al. Mutations in the Ras-Raf Axis underlie the prognostic value of CD133 in colorectal cancer. Clin Cancer Res, 2012;18:3132-41.

[27] Cheng Z, Li X, Ding J. Characteristics of liver cancer stem cells and clinical correlations. Cancer Lett, 2016;379:230-8.

[28] Banerjee S, Nomura A, Sangwan V, et al. CD133+ tumor initiating cells in a syngenic murine model of pancreatic cancer respond to Minnelide. Clin Cancer Res, 2014;20:2388-99.

[29] Jung YS, Vermeer PD, Vermeer DW, et al. CD200: Association with cancer stem cell features and response to chemoradiation in head and neck squamous cell carci- noma. Head Neck, 2015;37:327-35.

[30] Takaishi S, Okumura T, Tu S, et al. Identification of gastric cancer stem cells using the cell surface marker CD44. Stem Cells, 2009;27:1006-20.

[31] Jiang J, Zhang Y, Chuai S, et al. Trastuzumab (herceptin) targets gastric cancer stem cells characterized by CD90 phenotype. Oncogene, 2012;31:671-82.

[32] Park J, Kim SY, Kim HJ, et al. A reciprocal regulatory circuit between CD44 and FGFR2 via c-myc controls gastric cancer cell growth. Oncotarget, 2016;7:2867083.

[33] Zhang C, Li C, He F, et al. Identification of CD44+CD24+ gastric cancer stem cells. J Cancer Res Clin Oncol, 2011;137:1679-86.

[34] Chen T, Yang K, Yu J, et al. Identification and expansion of cancer stem cells in tumor tissues and peripheral blood derived from gastric adenocarcinoma patients. Cell Res, 2012;22:248-58.

[35] Nishii T, Yashiro M, Shinto O, et al. Cancer stem celllike SP cells have a high adhesion ability to the peritoneum in gastric carcinoma. Cancer Sci, 2009;100:1397402.

[36] Najafzadeh N, Mazani M, Abbasi A, et al. Low-dose all-trans retinoic acid enhances cytotoxicity of cisplatin and 5-fluorouracil on CD44(+) cancer stem cells. Biomed Pharmacother, 2015;74:243-51.

[37] Ju X, Jiao X, Ertel A, et al. V-Src oncogene induces Trop2 proteolytic activation via cyclin D1. Cancer Res, 2016;76(2):6723-34.

[38] Trerotola M, Rathore S, Goel HL, et al. CD133, Trop-2 and alpha2betal integrin surface receptors as markers of putative human prostate cancer stem cells. Am J Transl Res, 2010;2:135-44.

[39] Lin H, Huang JF, Qiu JR, et al. Significantly upregulated TACSTD2 and Cyclin D1 correlate with poor prognosis of invasive ductal breast cancer. Exp Mol Pathol, 2013;94:73-8.

[40] Zhao W, Ding G, Wen J, et al. Correlation between Trop2 and amphiregulin coexpression and overall survival in gastric cancer. Cancer Med, 2017;6(5):9941001

[41] Li Z, Jiang X, Zhang W. TROP2 overexpression promotes proliferation and invasion of lung adenocarcinoma cells. Biochem Biophys Res Commun, 2016;470:197204.

(Received 03 May 2017, Revised 20 May 2017, Accepted 29 May 2017) 